\title{
Surface plasmon resonance sensor based on a planar polychromatic OLED light source
}

\author{
Jörg Frischeisen*, Nils Reinke ${ }^{\dagger}$, Cornelia Ostermayr, Jürgen Neumann, Stefan Nowy, Wolfgang \\ Brütting \\ University of Augsburg, Institute of Physics, Experimental Physics IV, Universitätsstraße 1, 86159 \\ Augsburg, Germany
}

\begin{abstract}
We present a novel surface plasmon resonance (SPR) sensor based on an integrated planar and polychromatic light source. The sensor comprises an organic light emitting diode (OLED) and a metallic sensing layer located on opposite sides of a glass prism. We successfully fabricated and tested prototype sensors based on this approach by the use of different prism geometries and OLEDs with blue, green and red emission color. We investigated the angular and wavelength dependent SPR dispersion relation for sensing layers consisting of silver and gold of different thicknesses in contact with air. Further on we demonstrated the sensor function by real time monitoring of temperature changes inside an adjacent water reservoir as well as by recording the dissolving process of sodium chloride in water. This shows that the configuration can in principle be used for bio-sensing applications.

The presented technique offers the advantage that there is no necessity to couple light from external bulky sources such as lasers or halogen lamps into the sensing device which makes it particularly interesting for miniaturization. The presented SPR configuration can be monolithically integrated on one common substrate. Furthermore it is compatible with the planar glass light pipe platform for SPR sensing and the two-color approach for the determination of the thickness and the dielectric constant of thin films in a single experiment.
\end{abstract}

Keywords: Surface plasmon resonance, Optical sensor, Chemical sensor, Organic light emitting diode

\section{INTRODUCTION}

Surface plasmon resonance (SPR) sensors are based on electromagnetic surface plasmon waves (SPs) traveling at the interface between a dielectric and a metallic layer to detect changes of the refractive index of the dielectric close to the metal layer ${ }^{1}$. The excitation of surface plasmons is usually accomplished in the regime of attenuated total reflectance when p-polarized light is incident on a metal-coated glass prism (Kretschmann's geometry) ${ }^{2}$. The SPR can be observed as a minimum of the reflectivity of the metal either as a function of the incidence angle or the wavelength. Thereby changes of the refractive index in the medium adjacent to the metal film can be detected by shifts of the SPR position ${ }^{2,3}$. Due to the extreme sensitivity to small variations in the refractive index of the probed medium, SPR sensors are ideally suited for a variety of applications including the non-invasive characterization of the optical constants and the thickness of thin films ${ }^{4}$, monitoring of adsorption processes at metal surfaces ${ }^{5}$, the real time and label-free analysis of biospecific interactions ${ }^{6-8}$ or for the determination of the concentration of a material within a solvent ${ }^{9}$.

In recent years there has been growing commercial interest in SPR sensors ${ }^{5,10,11}$, especially through the development of compact devices. Miniaturized SPR sensors have been reported by several authors: Manuel and coworkers presented a robust SPR sensor based on a special prism coupling technique ${ }^{12}$. Foster and coworkers used a configuration which excites SPs by a convergent beam of monochromatic light ${ }^{13}$. However, the light source still remains a stumbling block in the development of small SPR sensors. Usually, SPR spectroscopy relies on bulky light sources with high power consumption, such as lasers or halogen lamps. There have been reports on the replacement of the traditional light sources by inorganic light emitting diodes (LEDs) ${ }^{14,15}$. A big drawback of these methods is that the fabrication of inorganic LEDs requires epitaxial techniques and crystalline substrates. Therefore, an additional molding step or coupling by optical components is necessary for the integration of inorganic LEDs into SPR sensors. This step limits the possibilities

\footnotetext{
* Corresponding author: joerg.frischeisen@physik.uni-augsburg.de

† Present address: Institute for Computational Physics, Zürcher Hochschule der Angewandten Wissenschaften, PO Box 805,8401 Winterthur, Switzerland
}

Optical Sensors 2008, edited by Francis Berghmans, Anna Grazia Mignani, Antonello Cutolo, Patrick P. Meyrueis, Thomas P. Pearsall, Proc. of SPIE Vol. 7003, 70031B, (2008) · 0277-786X/08/\$18 · doi: 10.1117/12.780522 
of miniaturization of the sensor device. Consequently, direct assembling of an integrated light source onto a monolithical SPR sensor device is desirable for the next step in sensor miniaturization.

In contrast to their inorganic counterparts, organic light emitting diodes (OLEDs) can be fabricated on almost any surface with an adequate smoothness as their growth does not require epitaxy. They are area emitters with a spectrally extended emission and are considered as promising candidates for general illumination and display technology. However, there have also been suggestions for other niche applications in recent years. Bürgi and coworkers developed an optical proximity and touch sensor based on monolithically integrated polymer light emitting diodes and polymer photodiodes ${ }^{16}$. Wang and coworkers reported that polymer light emitting diodes and polymer photodetectors can be integrated on disposable polydimethylsiloxane microfluidic flowcells ${ }^{17}$.

In this work we demonstrate a novel SPR sensor on the basis of an integrated OLED light source ${ }^{18}$. The sensor includes a metallic sensing layer and an organic light emitting diode located on opposite sides of one common prism. The light emitted by the OLED leaves the prism after reflection at the sensing layer. We analyze the spectral and angular dependent intensity of the reflected light for sensing layers consisting of silver and gold with different thicknesses. Further on we demonstrate the sensor function by real time monitoring of temperature changes inside an adjacent water reservoir and by recording the dissolving process of sodium chloride $(\mathrm{NaCl})$ in water.

\section{METHODOLOGY}

\subsection{Fabrication of organic light emitting diodes and metallic sensing layers}

We used three OLEDs with different emission spectra as the SPR light source in our experiments. All OLEDs were fabricated on pre-cleaned commercial glass substrates covered with indium tin oxide (ITO) of $140 \mathrm{~nm}$ thickness (Merck Display Technologies, Type 859) as the anode. We deposited approximately $30 \mathrm{~nm}$ of the hole injection layer Poly(3,4ethylenedioxythiophene) poly(styrenesulfonate) (PEDOT:PSS) by spin coating and all the other organic layers and metallic electrodes by thermal evaporation in a high vacuum system. The blue OLED comprises $40 \mathrm{~nm}$ of the hole transport material 2,2',7,7'-tetrakis-(N,N-diphenylamino)-9,9'-spirobifluoren (S-TAD), $30 \mathrm{~nm}$ of the blue emitter 4,4'bis(2,2'-diphenylvinyl)-1,1'-spirobiphenyl (S-DPVBi), and $20 \mathrm{~nm}$ of the electron transporter Tris-(8-hydroxyquinoline) aluminum $\left(\mathrm{Alq}_{3}\right)$. The green OLED consists of $80 \mathrm{~nm} \mathrm{S-TAD}$ and $80 \mathrm{~nm}$ of the electron transporter and green emitter $\mathrm{Alq}_{3}$. Finally, the red OLED is made up of the same materials as the green OLED apart from a $20 \mathrm{~nm}$ wide zone of the $\mathrm{Alq}_{3}$ layer at the interface to S-TAD being doped with the red dye 4-dicyanomethylene-2-methyl-6-(pdimethylaminostyryl)-4H-pyran (DCM). The ratio of $\mathrm{Alq}_{3}: \mathrm{DCM}$ in the doped layer is $20: 1$ by weight. For the next $30 \mathrm{~nm}$ $\mathrm{Alq}_{3}$ the deposition rate of DCM was linearly reduced to zero. All OLEDs had a $10 \mathrm{~nm}$ thick Ca layer covered by 120 to $150 \mathrm{~nm} \mathrm{Al}$ as the cathode. Finally, we encapsulated the devices by a cover glass and UV-curing epoxy resin. As metallic sensor films, we thermally evaporated silver and gold with different thicknesses on pre-cleaned glass substrates. For the measurements in aqueous environment, we removed a part of a cuvette and attached the metal sensing film to it so that about $5 \mathrm{ml}$ solvent can be added.

\subsection{Experimental setup}

The experimental arrangement is shown in Fig. 1. For SPR measurements at the metal/air interface two right-angle prisms were connected by index matching gel to form one parallelogram and the OLED and the metallic sensing layer are attached to opposite sides of the prism by index matching gel, cf. Fig. 1(a).

(a)

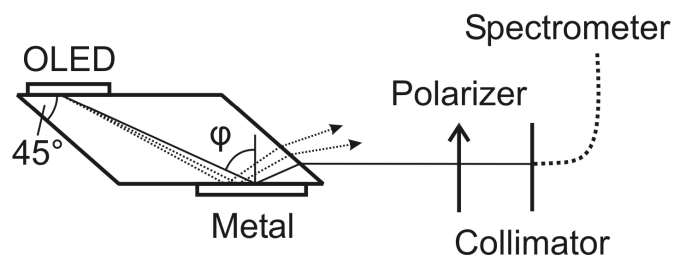

(b)

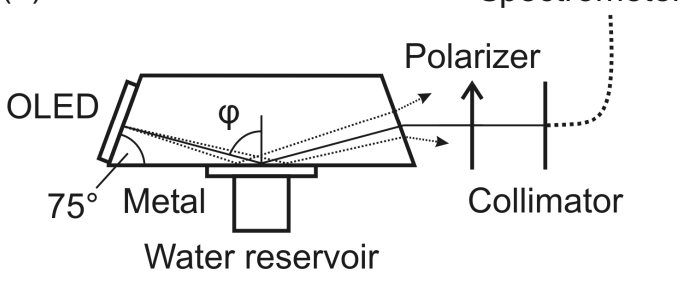

Fig. 1. Experimental setup: (a) for measurements in air an organic light emitting diode and a metallic sensor film are located on opposite sides of a glass prism which is mounted on a motorized rotation stage. The reflected light leaves through the edge of the prism and passes a linear polarizing filter before it is focused by a collimating lens onto an optical fiber guiding it to a spectrometer. (b) For measurements in aqueous environment the OLED and the metal film are attached to the edges of a trapezoidal plexiglass prism forming an angle of about 75 degrees towards the prism base. 
For SPR measurements in aqueous environment the OLED is attached to the left edge of a trapezoidal plexiglass prism and the metallic sensing layer is located at the base of the prism with an angle of approximately 75 degrees between them, as shown in Fig. 1(b). Inside the glass prism the OLED irradiates the metallic sensing layer with polychromatic light under various angles of incidence. The reflected light leaves through the edge of the prism and passes a linear polarizing filter so that only the p-polarized fraction is detected. A collimating lens focuses the light onto an optical fiber guiding it to a calibrated CCD spectrometer (Avantes AvaSpec-2048).

For the measurement of the plasmon dispersion relation the prism was mounted on a motorized computer controlled rotation stage in such a way that the illuminated metal film was located on the axis of rotation. The angular dependence of the reflectivity of the metal film was detected by rotating the turntable in steps of 0.1 degree in the measurements with the red and green OLED and 0.2 degree for the blue OLED. A spectrum of the reflected light was recorded after each angle step. Additionally, the refraction of the light at the prism/air interface must be taken into account in order to determine the angle of incidence under which the light of the OLED impinges on the metal film.

In order to obtain the p-polarized reflectivity a reference measurement was performed for each experiment. Thereby the metallic sensor film was replaced by a glass substrate without a metallic layer and again a spectrum was recorded for each step. In the measured wavelength regime, total reflection occurs at the glass/air interface and the intensity of the reflected light is equal to the incident intensity. Hence by dividing the measured spectrum by the reference, one can obtain the p-polarized reflectivity for each angle. For the measurement over time in aqueous environment, which was performed at a fixed angle of incidence, the measured spectra were divided by one reference spectrum that was recorded under the same angle.

Fig. 2 shows the emission spectra of the blue, green and red OLEDs recorded in the normal direction towards the substrate plane. The full-width at half-maximum (FWHM) of the three OLEDs is about $20 \%$ of the peak wavelength. The blue, green and red OLEDs have peak wavelengths of $465 \mathrm{~nm}, 530 \mathrm{~nm}$ and

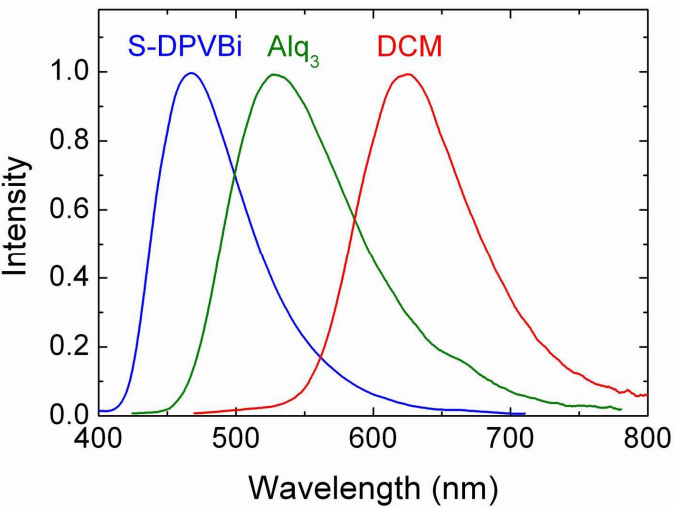

Fig. 2. OLED emission spectra normal to the substrate using three different organic emitters as described in the text. $625 \mathrm{~nm}$, respectively, and cover almost the whole visible spectrum.

The active area of all OLED pixels is $10 \mathrm{~mm}$ long and $0.5 \mathrm{~mm}$ wide, so that the OLED can be regarded as a linear light source. Compared to an OLED with a quadratic active area this geometry has the advantage that due to the small width all light originates from almost the same angular position while the large length increases the intensity of the detected light. A constant current source (Keithley, Type 220) drives the OLED with a current of $0.6 \mathrm{~mA}$ which corresponds to a moderate current density of $12 \mathrm{~mA} / \mathrm{cm}^{2}$ so that there should be no considerable degradation. If an OLED degrades over time the voltage required for a constant current usually increases. In our setup the voltage was monitored with a separate instrument (Keithley, Type 196) and no degradation was observable. Hence the light output of the OLEDs can be assumed constant over time.

\section{RESULTS AND DISCUSSION}

\subsection{Metal/air interface}

The following equation describes the dispersion relation for surface plasmons at the border of two half spaces ${ }^{2}$ :

$$
k_{\mathrm{x}}=\frac{\omega}{c} \sqrt{\frac{\varepsilon_{1}(\omega) \varepsilon_{2}}{\varepsilon_{1}(\omega)+\varepsilon_{2}}} .
$$

Therein $k_{\mathrm{x}}$ and $\omega$ are the complex in-plane wave vector and the angular frequency of the surface plasmon, respectively. $\varepsilon_{1}(\omega)=\varepsilon_{1}^{\prime}(\omega)+i \varepsilon^{\prime \prime}{ }_{1}(\omega)$ denotes the complex dielectric function of the metallic sensing layer and $\varepsilon_{2}$ the dielectric constant of the environment. If we assume besides a real $\omega$ and $\varepsilon_{2}$ that $\varepsilon^{\prime \prime}{ }_{1}(\omega)<\left|\varepsilon_{1}^{\prime}(\omega)\right|$, we obtain the following equation for the real component of the in-plane wave vector $k_{\mathrm{x}}^{\prime}$ : 


$$
k_{\mathrm{x}}^{\prime}=\frac{\omega}{c} \sqrt{\frac{\varepsilon_{1}^{\prime}(\omega) \varepsilon_{2}}{\varepsilon_{1}^{\prime}(\omega)+\varepsilon_{2}}} .
$$

For a real $k_{\mathrm{x}}^{\prime}$ one needs $\varepsilon_{1}^{\prime}(\omega)<0$ and $\left|\varepsilon_{1}^{\prime}(\omega)\right|>\varepsilon_{2}$, which can be fulfilled in a metal or a doped semiconductor. The imaginary part of the wave vector $k^{\prime \prime}{ }_{x}$ determines the absorption of the surface plasmon mode. Using the fact that the in-plane wave vector is continuous across the glass/metal interface, i.e. $k_{\mathrm{x}}^{\prime}=(\omega / c) n_{\text {Substrate }} \sin \varphi$ with $n_{\text {Substrate }}$ being the refractive index of the glass substrate, we find the following resonance condition for the incident angle $\varphi$ from the glass side:

$$
\sin \varphi=\frac{1}{n_{\text {Substrate }}} \sqrt{\frac{\varepsilon_{1}^{\prime}(\omega) \varepsilon_{2}}{\varepsilon_{1}^{\prime}(\omega)+\varepsilon_{2}}}=\frac{1}{n_{\text {Substrate }}} \sqrt{\frac{\varepsilon_{1}^{\prime}(\lambda) \varepsilon_{2}}{\varepsilon_{1}^{\prime}(\lambda)+\varepsilon_{2}}} .
$$

For the evaluation of Eq. (3), we use the complex dielectric functions from ellipsometric measurements. Fig. 3 shows the dispersion relation of surface plasmons at $\mathrm{Ag} / \mathrm{air}$ and $\mathrm{Au} / \mathrm{air}$ interfaces obtained by Eq. (3). Note that this equation assumes a semi-infinite metal layer, thus it can be used only as an approximation for thin metal films. In Fig. 3 it can be observed that the SPR feature at the Au/air interface moves to very high angles for wavelengths below $520 \mathrm{~nm}$. In this region the absolute value of the real part of the dielectric function of $\mathrm{Au}$ approaches the dielectric constant of the environment $\left(\left|\varepsilon_{1}^{\prime}(\omega)\right| \rightarrow \varepsilon_{2}\right)$. Therefore the denominator in Eq. (3) approaches zero, thus increasing the angle $\varphi$. For wavelengths below $475 \mathrm{~nm}$ the SPR minimum disappears completely because $\left|\varepsilon_{1}^{\prime}(\omega)\right|<1$, thus the radicand turns negative and the condition for the excitation of SPs can not be fulfilled anymore. As a consequence, SPs can not be excited at the $\mathrm{Au} / \mathrm{air}$ interface in the blue wavelength range. In contrast, at the $\mathrm{Ag} /$ air interface the SP dispersion can still be observed at these wavelengths because $\left|\varepsilon_{1}^{\prime}(\omega)\right|$ is considerably larger than unity

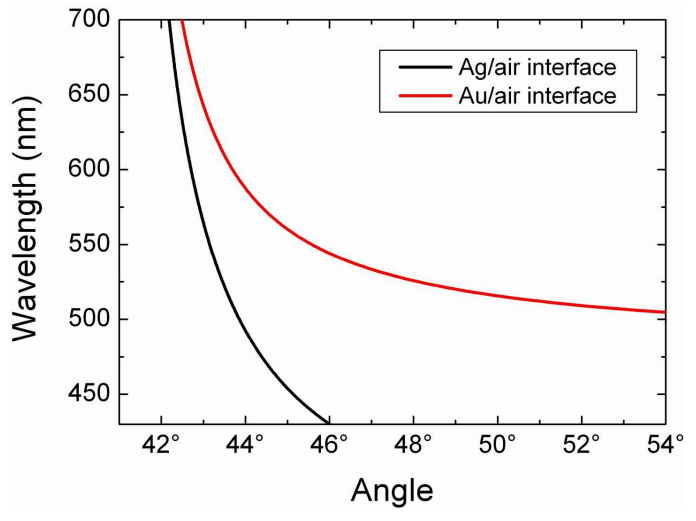

Fig. 3. Dispersion relation of surface plasmons at $\mathrm{Ag} / \mathrm{air}$ and $\mathrm{Au} / \mathrm{air}$ interfaces according to Eq. (3). For decreasing wavelengths the SPR minimum moves to higher angles. even for a wavelength of $400 \mathrm{~nm}$.

Fig. 4 shows the measured and simulated p-polarized reflectivity at the interface of a $49 \mathrm{~nm}$ thick silver film and air obtained over a broad spectral range using three different OLEDs. The silver thickness was monitored with a quartz
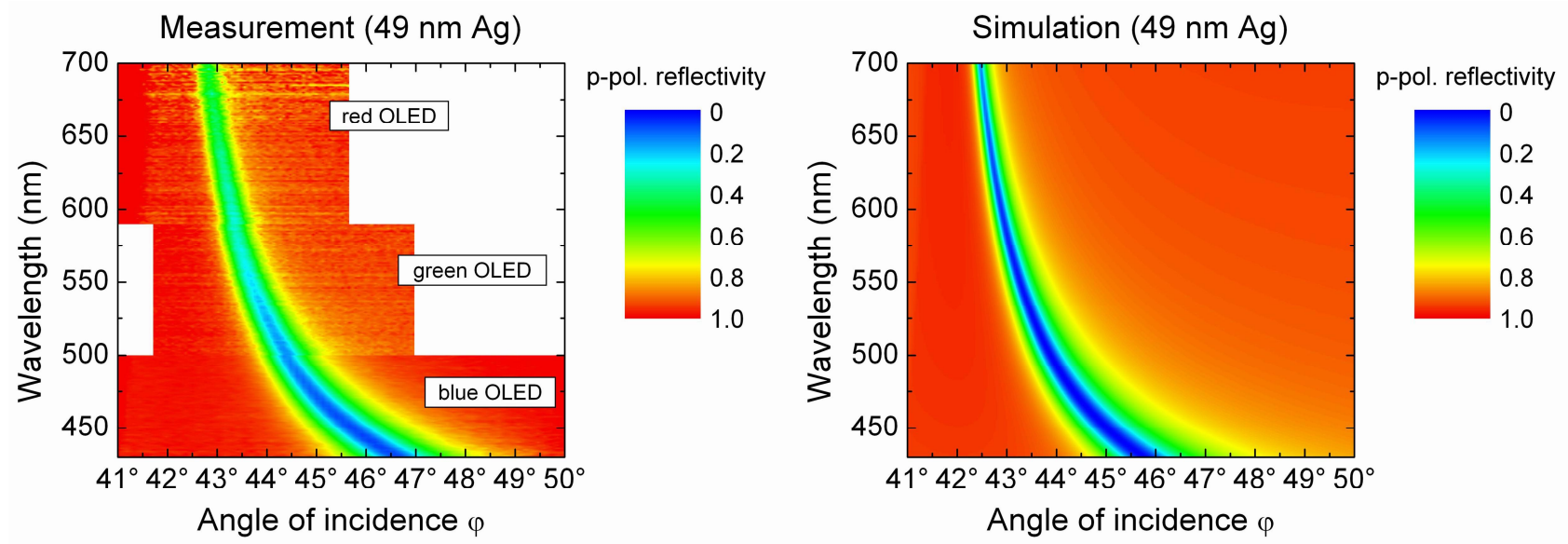

Fig. 4. Measurement (left) and simulation (right) of the spectral and angular resolved p-polarized reflectivity of a $49 \mathrm{~nm}$ thick silver film exposed to air. Regions of low reflectivity indicate resonant coupling to the surface plasmon. 
microbalance during the evaporation and subsequently controlled with profilometry (DekTak). The simulation was carried out with the commercial software ETFOS (Fluxim AG, Switzerland) using optical constants from ellipsometric measurements. At the chosen silver thickness it is expected that the resonance condition for the excitation of SPs is ideal and the reflectivity should drop to virtually zero over a broad wavelength range as can be seen in the simulation. The observed angular dependence of the reflectivity minimum is in excellent agreement with the simulation. Slight deviations in the angular position can be attributed to small errors in the optical constants used for the simulation. For wavelengths larger than $550 \mathrm{~nm}$ the full width at half maximum of the simulated reflectivity minimum is less than 0.5 degrees and can not be resolved anymore due to the finite angular resolution of the experimental setup. Therefore the measured intensity does not reach zero anymore. However, the angular position of the minimum still coincides well with the simulation.

To illustrate the influence of the metal thickness we have repeated the experiment with a reduced silver thickness of $33 \mathrm{~nm}$. The resulting measurement and the appropriate simulation are shown in Fig. 5. Again the measured reflectivity agrees well with the theoretical prediction.
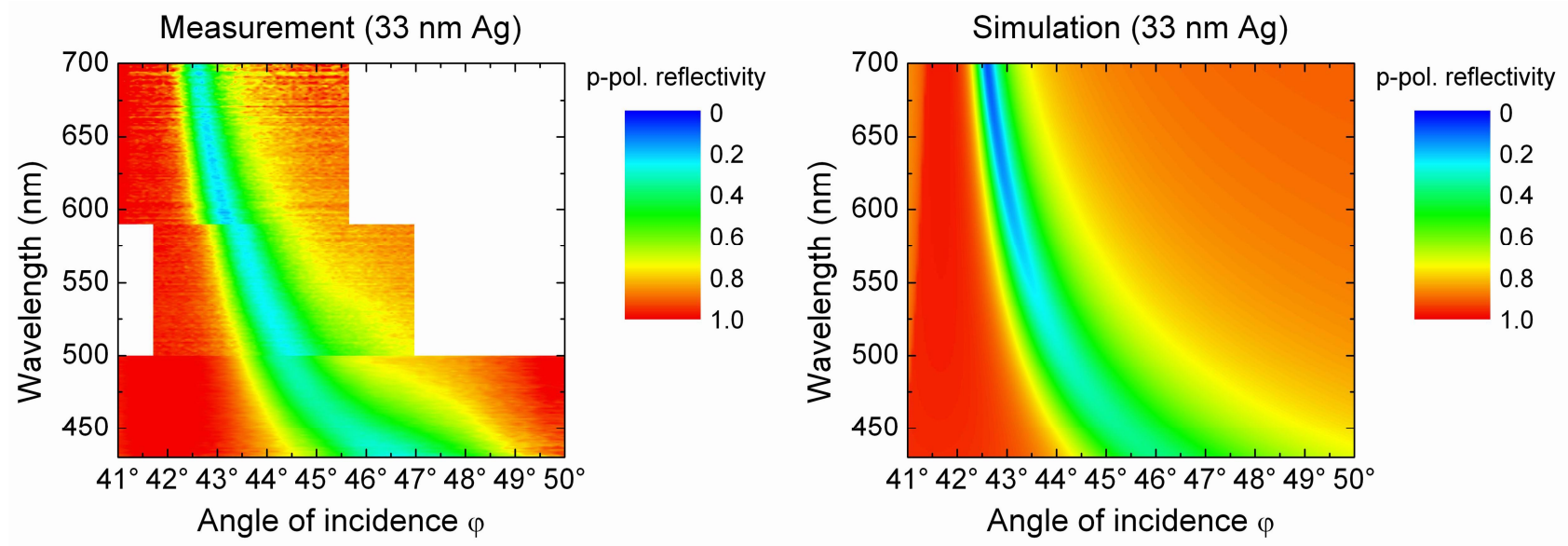

Fig. 5. Measured (left) and simulated (right) p-polarized reflectivity of a $33 \mathrm{~nm}$ thick silver film exposed to air.

Comparing the reflectivity of the two silver films with different thicknesses it is obvious that the minimum is less distinct and also significantly broader in the case of $33 \mathrm{~nm}$ silver. This illustrates the importance of choosing the optimum silver thickness which depends strongly on the wavelength and the medium adjacent to the metal film. The fact that the measured reflectivity is in accordance with the simulation reveals that simulations can be applied to predict the optimum silver thickness. Generally, approximately $50 \mathrm{~nm}$ thickness is best for measurements at the Ag/air interface in the visible wavelength regime. If the thickness differs from the optimum value, zero reflectivity can not be obtained. In detail, thinner films result in a broader and less pronounced minimum as shown in the $33 \mathrm{~nm} \mathrm{Ag}$ measurement. Simulations show that film thicknesses larger than the optimum value result in less distinct minima as well; however, the width does not increase significantly in this case.

By comparison of the angular position of the minimum in Figs. 3, 4 and 5 it is noticeable that the position slightly shifts to smaller angles with increasing silver thickness, being highest for the case in Fig. 3 which was calculated for a semiinfinite silver layer using Eq. (3). Thus Eq. (3) is not exactly valid for thin silver films but nevertheless can be used as a good approximation.

In addition to the experiments at a $\mathrm{Ag} /$ air interface, we have performed measurements at a $\mathrm{Au} /$ air interface in order to illustrate the influence of the material that is used as sensing layer. The results for two gold thicknesses of $49 \mathrm{~nm}$ and $27 \mathrm{~nm}$ are shown in Fig. 6. The overall agreement between experiment and simulation is again excellent. As expected from Fig. 3 the dispersion relation of the SPs is located at higher angles compared to silver. In addition, the SPR feature is much broader in the case of gold. For small wavelengths the SPR feature disappears completely and the reflectivity at the $\mathrm{Au} / \mathrm{air}$ interface is low over the whole range of angles because the frequency of light approaches the plasma frequency of gold.

The optimum thickness for gold amounts to about $45 \mathrm{~nm}$ in the visible wavelength regime, but $49 \mathrm{~nm}$ still yields an excellent result and the minimum drops to almost zero. Again it is observable that the SPR feature is less pronounced and broader for a $27 \mathrm{~nm}$ Au layer compared to the thicker one. 

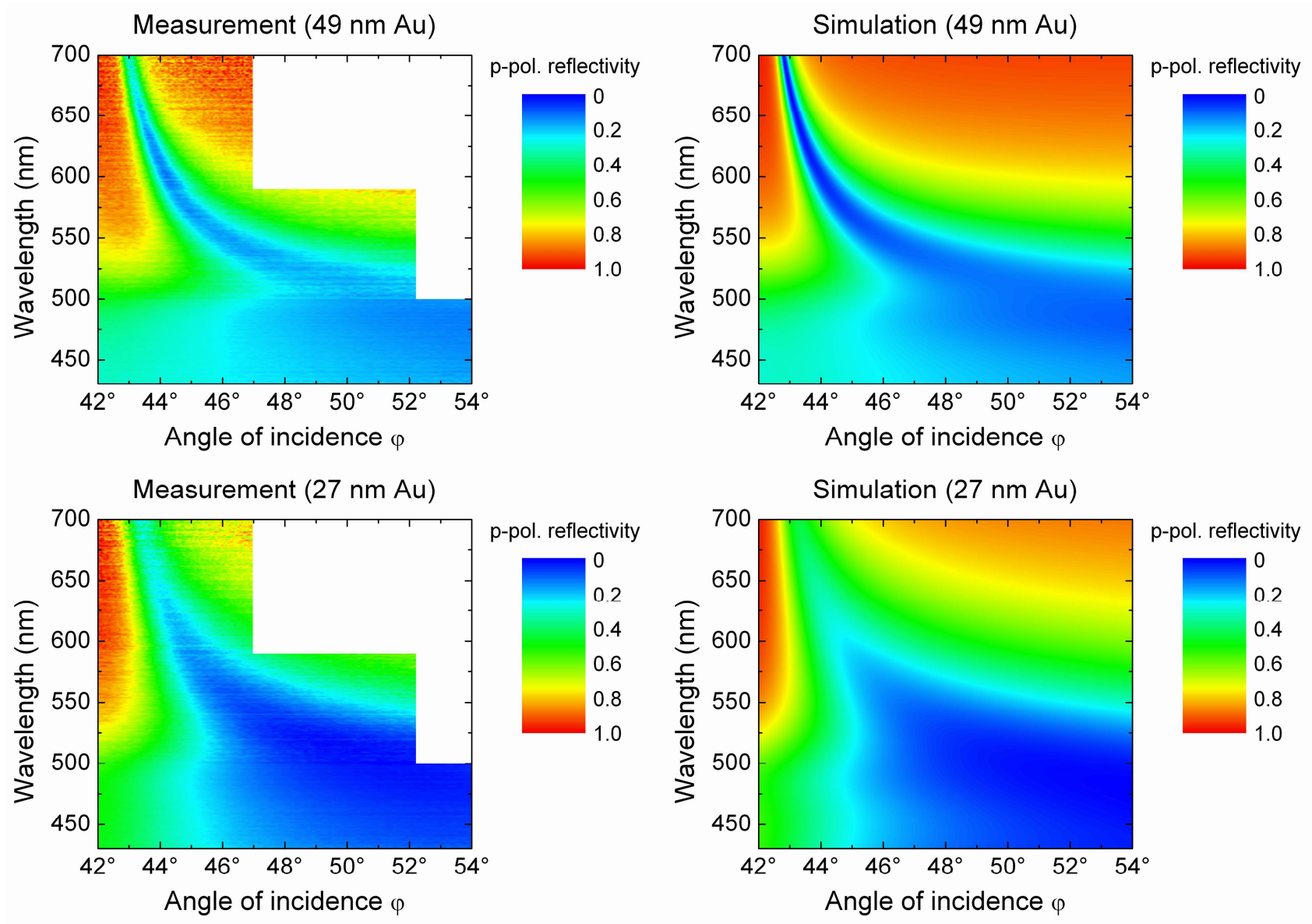

Fig. 6. Top: Measurement (left) and simulation (right) of the spectral and angular resolved p-polarized reflectivity of a $49 \mathrm{~nm}$ thick gold film exposed to air. Bottom: As above except of a gold thickness of $27 \mathrm{~nm}$.

The experiments performed at $\mathrm{Ag} / \mathrm{air}$ and $\mathrm{Au} /$ air interfaces have demonstrated the basic functionality of the novel sensor. The perfect agreement between measurements and simulations makes it possible to predict the angular position for all wavelengths even for media different than air provided that the optical constants are known. This is advantageous for the measurements in aqueous environments shown in the following sections.

\subsection{Real time monitoring of temperature changes}

To demonstrate the sensor capability of performing measurements in real time we monitored temperature changes and the dissolving process of $\mathrm{NaCl}$ in a water reservoir adjacent to the metallic sensing layer. For this purpose we evaporated $49 \mathrm{~nm} \mathrm{Au}$ on an approximately $2 \mathrm{~nm}$ thick Ti undercoating layer to increase the adherence of gold on the glass substrate in contact with the aqueous environment. We used the red OLED as a light source in these experiments. Simulations predict that the SPR feature shifts to a higher angle of about 75 degrees if the Au layer is in contact with water. Thus, we have designed an optimized trapezoidal plexiglass prism so that the light from the OLED is incident under approximately 75 degrees on the metal film. All experiments were performed at room temperate $\left(20^{\circ} \mathrm{C}\right)$ and the changes over time at a constant angle of incidence were monitored. By increasing the temperature of water, it is expected that the spectral position of the minimum shifts to smaller wavelengths, because the refractive index of water is reduced with increasing temperature ${ }^{19}$.

The measurement of the reflectivity as a function of wavelength during the change of the water temperature is shown in Fig. 7. For the first five minutes the cuvette next to the gold film was empty, thus we measured the reflectivity of a $\mathrm{Au} /$ air interface at 75 degrees angle of incidence which is far away from the resonant position. Therefore no minimum can be observed and the reflectivity of the gold film is very high over the whole wavelength range. 


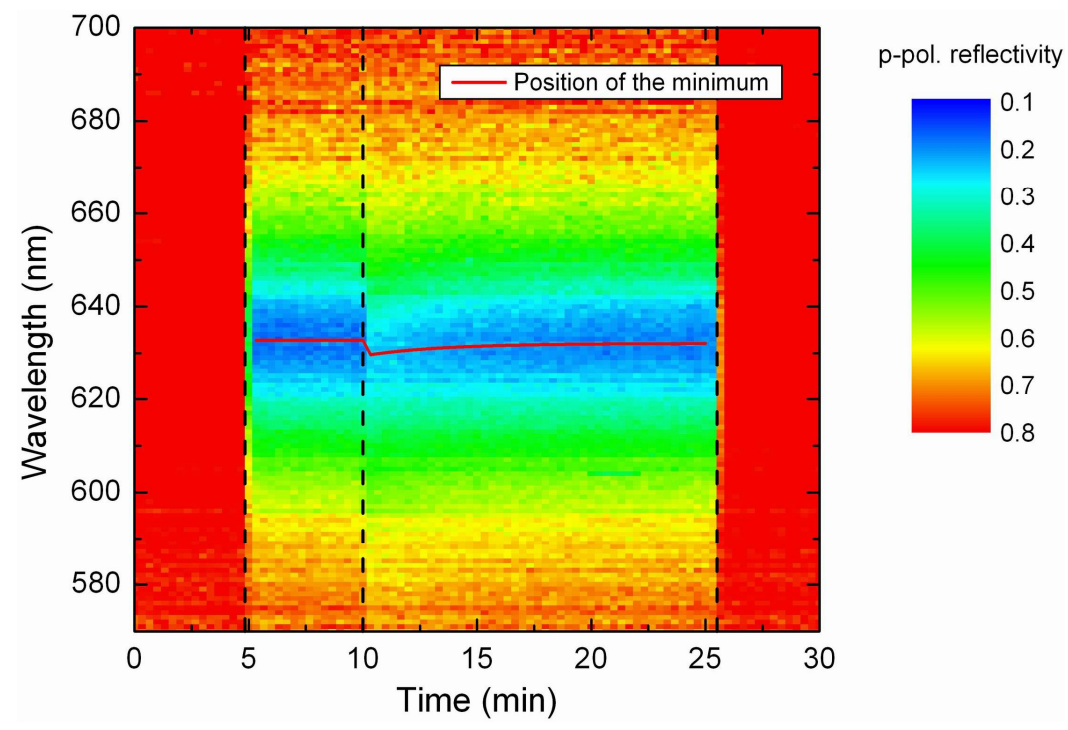

Fig. 7. Measurement of the reflectivity change over time of a $49 \mathrm{~nm}$ Au film adjacent to a water reservoir at an incidence angle of 75 degrees. Initially, the cuvette is empty, hence we measure the reflectivity of a Au/air interface and no SPR feature is observable at this angle. After five minutes we added $1.5 \mathrm{ml}$ water with a temperature of $20^{\circ} \mathrm{C}$. Five minutes later we added $3 \mathrm{ml}$ water with a temperature of $80{ }^{\circ} \mathrm{C}$ which induces a shift of the minimum to smaller wavelengths and a subsequent relaxation as the water cools down. The water was removed after 25 minutes.

After five minutes we added about $1.5 \mathrm{ml}$ water with a temperature of $20^{\circ} \mathrm{C}$. This is sufficient to cover the gold film completely with water. Instantly a pronounced SPR minimum appears with the lowest reflectivity at about $633 \mathrm{~nm}$ wavelength. The spectral position of the minimum remains constant until $3 \mathrm{ml}$ water with a temperature of $80{ }^{\circ} \mathrm{C}$ is added after ten minutes. The addition of hot water causes the minimum to shift to a lower wavelength of approximately $629 \mathrm{~nm}$ which basically agrees with the expected behavior. After 25 minutes we removed the water inside the cuvette which causes the SPR feature to disappear and the reflectivity goes back to the initial value.

Fig. 8 shows the temperature that we have deduced from the spectral position of the minimum for each time step. The spectral position was assumed constant for the first five minutes and after the addition of hot water an exponential decay was fitted to the position of the minimum (red line in Fig. 8). Thus we know the spectral position of the minimum for each time step. We identify this position with the point of optimum excitation of SPs and hence we can use Eq. (3) to determine the dielectric constant $\varepsilon_{2}$ of the adjacent water reservoir. We use the position of the minimum in the first five minutes to define the constant $n_{\text {Substrate }} \sin \varphi$ because the angle of incidence is not known precisely. We find a value of 1.429 which is close to the value of 1.439 that is expected for $\varphi=75^{\circ}$ and

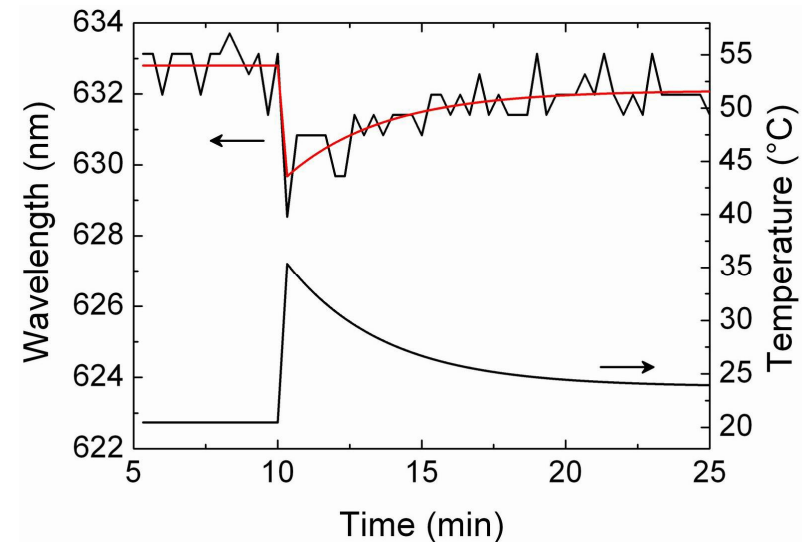

Fig. 8. Extracted and smoothed spectral position of the minimum in Fig. 7 and deduced temperature for each time step. The red line indicates a fit to the position of the minimum.

$n_{\text {Substrate }}=1.49$ (plexiglass). Then we are able to determine

$\varepsilon_{2}$ with Eq. (3) and therewith the refractive index of the adjacent water reservoir $n_{2}=\sqrt{\varepsilon_{2}}$ for each time step. Finally, we can derive the temperature of the water reservoir through the correlation between temperature and refractive index of water ${ }^{19}$, which can be well described by a linear approximation in the range between $20^{\circ} \mathrm{C}$ and $40{ }^{\circ} \mathrm{C}$. Actually, this correlation is valid exactly only for a wavelength of $632.8 \mathrm{~nm}$ as in the table in Ref. 19, but using this correlation also for wavelengths between $629 \mathrm{~nm}$ and $633 \mathrm{~nm}$ causes an error in the determined refractive index which is more than an order of magnitude smaller and thus negligible compared to the measured refractive index change. 
It is expected that the temperature reaches a value of $60{ }^{\circ} \mathrm{C}$ after the addition of the hot water. Our measurement indicates a lower temperature which can be explained because the hot water cools down rapidly in contact with the cuvette and the glass prism. Every measuring step takes 15 seconds, thus we measure only the average temperature for each step and we can not resolve the highest temperature that occurs directly after the addition of the hot water. However, we have demonstrated that a temperature change of the solvent adjacent to the metallic sensing layer can be clearly observed.

\subsection{Measurement of the dissolving process of sodium chloride}

SPR sensors are widely used to monitor biospecific interactions or to determine concentrations of biomolecules in a solvent. To demonstrate the feasibility of our sensor to detect changes in concentration in real time we have dissolved sodium chloride in a water reservoir adjacent to the same $49 \mathrm{~nm}$ gold film used in the previous experiment. It is expected that the spectral position of the minimum shifts to higher wavelengths with increasing $\mathrm{NaCl}$ concentration ${ }^{20}$. In Fig. 9 we show the reflectivity of a $\mathrm{Au} /$ water interface as a function of wavelength and time.

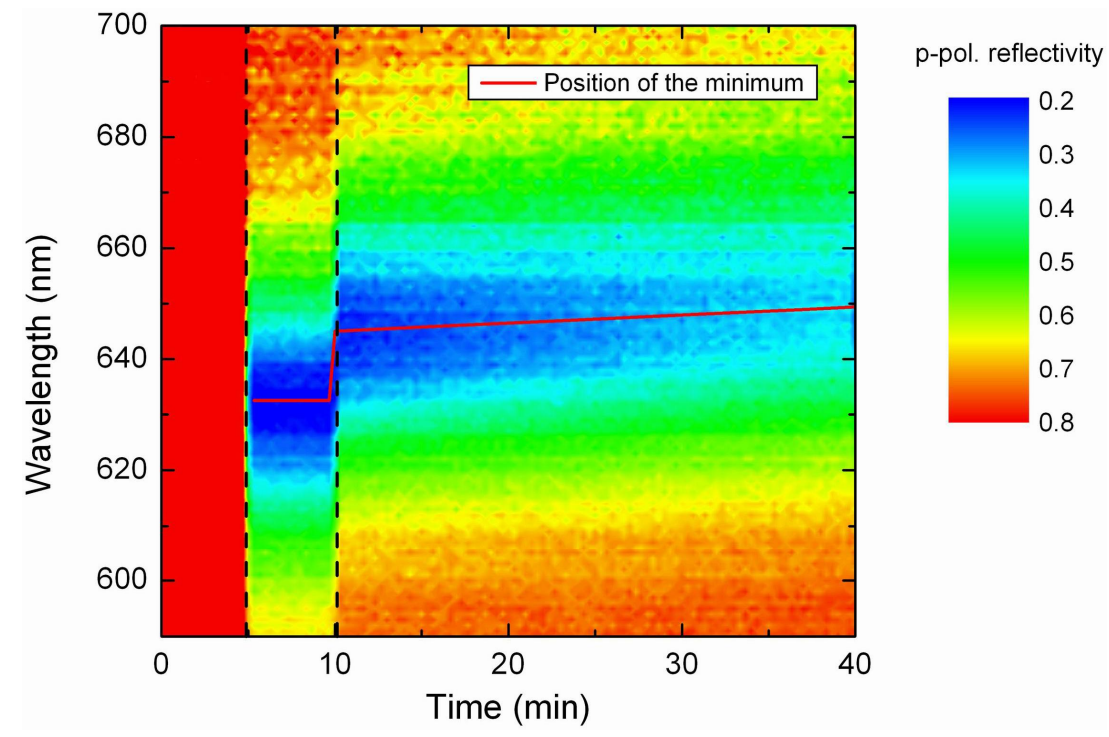

Fig. 9. Wavelength and time dependent reflectivity of a Au/water interface. The water reservoir is empty in the beginning. After five minutes we added $1.5 \mathrm{ml}$ water. Five minutes later we added $0.5 \mathrm{mg} \mathrm{NaCl}$ which causes an immediate shift of the spectral position of the minimum to higher wavelengths and a subsequent slow increase.

As in the measurement of the temperature change, we left the water reservoir empty for the first five minutes. Again there is no SPR feature observable and the reflectivity is high over the entire wavelength range. After adding $1.5 \mathrm{ml}$ water a pronounced minimum appears and the lowest reflectivity is again located at a wavelength of about $633 \mathrm{~nm}$. After ten minutes we added $0.5 \mathrm{mg} \mathrm{NaCl}$. A considerable fraction of the salt is dissolved immediately and causes an instant shift of the minimum position to $645 \mathrm{~nm}$. Subsequently, the minimum continues to shift to higher wavelengths as the remaining salt slowly dissolves.

To obtain the index of refraction of the solution from the spectral position of the SPR feature we used a refractive index of $n=1.3329$ for pure water for the position of the minimum between $\mathrm{t}=5 \mathrm{~min}$ and $10 \mathrm{~min}$. For $\mathrm{t}>10 \mathrm{~min}$ we made a linear fit to the spectral position of the minimum as shown in Fig. 10 and we calculated the index of refraction $n_{2}=\sqrt{\varepsilon_{2}}$ using Eq. (3). The increase of the refractive index

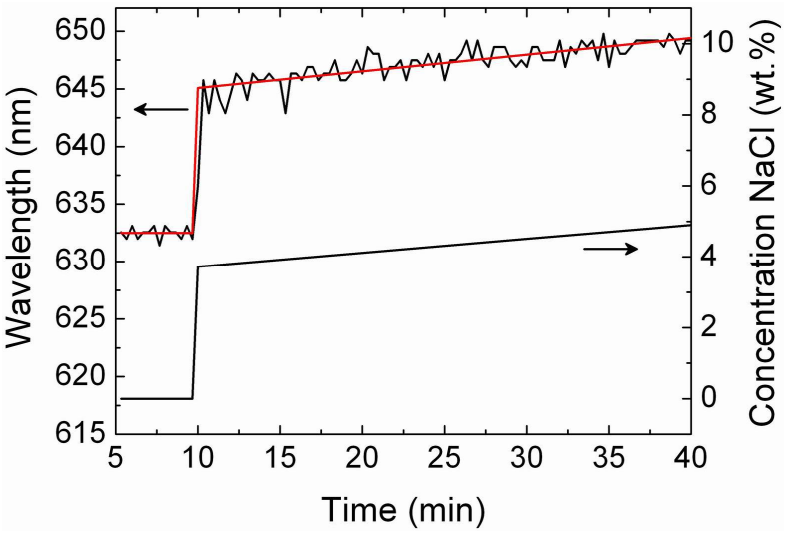

Fig. 10. Extracted and smoothed spectral position of the minimum in Fig. 9 and calculated concentration of $\mathrm{NaCl}$. The red line indicates a fit to the position of the minimum. 
with the concentration of dissolved sodium chloride ions $\mathrm{c}_{\mathrm{NaCl}}$ (in wt.\%) is well described by the following equation

$$
\mathrm{n}=0.00177 \mathrm{c}_{\mathrm{NaCl}}+1.3329
$$

which was derived from data in Ref. 20. Although this equation is only exact for one specific wavelength, we use it in the wavelength range between $633 \mathrm{~nm}$ and $650 \mathrm{~nm}$. The induced error in the determined refractive index is negligible compared to the measured change in refractive index. We used Eq. (4) to extract the concentration of sodium chloride in the solution as depicted in Fig. 10. Thirty minutes after the dissolving process has started, the concentration of sodium chloride reaches a value of approximately $5 \mathrm{wt} . \%$. The dissolving process could be increased by stirring. This might also prevent that the minimum gets less pronounced over time as can be observed in Fig. 9. It is likely that this behavior is caused by the fact that most of the salt sediments on the ground. Thus the water near the ground has a higher salt concentration than the water at the top and we measure the average of a mixture of different salt concentrations. However, we have shown that our novel sensor is capable of monitoring the concentration of dissolved $\mathrm{NaCl}$ in water in real time, thus the sensor also has the potential of measuring concentrations of other materials or biomolecules within a solvent.

\section{CONCLUSIONS}

We have demonstrated a novel SPR sensor based on the combination of a metallic sensing layer and an integrated OLED light source located on opposite sides of a prism. We have analyzed the spectral and angular dependent intensity of the reflected light for sensing layers consisting of silver and gold of different thicknesses and we have shown the importance of choosing the appropriate metal thickness. The measurements at metal/air interfaces are in excellent agreement with the simulations. Further on we have demonstrated that the sensor is capable of real time monitoring temperature changes inside an adjacent water reservoir. In addition, we have recorded the dissolving process of sodium chloride in water in real time. This shows that the configuration can in principle be used for bio-sensing applications like the label-free analysis of protein interactions or adsorption processes as well as for the determination of concentrations of a given material within a solvent.

The sensitivity of an SPR sensor is determined by the magnitude of the wavelength shift divided by the change in refractive index ${ }^{21}$. It is given in units of $\mathrm{nm} / \mathrm{RIU}$ (refractive index unit), where the difference between a refractive index of 1.4 and 1.3 is 0.1 RIUs. For our measurements the wavelength shifts approximately $20 \mathrm{~nm}$ if the refractive index changes by 0.01 , thus the sensitivity is $2000 \mathrm{~nm} / \mathrm{RIU}$. White and Fan emphasize that the sensor resolution, which characterizes the smallest possible spectral shift that can be accurately measured, is an equally important property of an SPR device ${ }^{21}$. In our setup the sensor resolution is approximately $1 \mathrm{~nm}$. The sensitivity and sensor resolution combine to form the detection limit of the device. For our setup, the detection limit is around $5 \times 10^{-4}$ RIU. These values can be enhanced by decreasing the width of the SPR feature, for instance by the utilization of optical multilayer-structures supporting symmetrically bound surface plasmons ${ }^{22}$. In addition, the sensor resolution can be further increased by operating the OLED at a higher voltage. In our measurements the OLED was driven with a current density of only $12 \mathrm{~mA} / \mathrm{cm}^{2}$. Furthermore, the usage of a white OLED could be favorable for covering a larger spectral range, hence making it possible to use the sensor in a wide range of applications. By contrast, a narrower emission spectrum might be advantageous for specific measurements in a small wavelength range. Due to the fact that the OLED has a spectrally extended emission it is not possible to achieve the same resolution as a device with a laser light source. However, we want to emphasize that in contrast to SPR devices based on a laser, our sensor offers the advantage that there is no necessity to couple light from external bulky sources into the device which makes the sensor particularly interesting for miniaturization. We anticipate that the presented SPR configuration can be monolithically integrated on one common substrate, thus making it unnecessary to use a prism. It is also particularly compatible with the planar glass light pipe platform for SPR sensing, reported by several authors ${ }^{1,23}$. An additional benefit of the usage of an OLED is that the active area can easily be adjusted to any shape required for a specific application. Finally, we want to mention that our sensor can in principle be used for the simultaneous determination of the thickness and dielectric constant of thin films. Because it is impossible to obtain both of these parameters from a single SPR experiment, Peterlinz and Georgiadis have proposed to use two lasers with different wavelengths in one experiment to measure both thin film quantities at the same time ${ }^{4}$. Due to the broad emission range of OLEDs it should be possible to realize this idea with only one OLED. However, even a second OLED could be placed directly adjacent to the first OLED, thus offering for example a simultaneous red and blue emission without reducing the tremendous potential for miniaturization.

Proc. of SPIE Vol. 7003 70031B-9 


\section{ACKNOWLEDGEMENTS}

We thank Christian Bergner for the ellipsometric measurement of complex refractive indices. We acknowledge financial support by the Elite Network of Bavaria through the international graduate school "Materials Science of Complex Interfaces" (J. Frischeisen and N. Reinke) and through the international graduate school "NanoBioTechnology" (J. Neumann) as well as OSRAM OS for providing us with some of the OLED materials.

\section{REFERENCES}

[1] J. Homola and S. S. Yee, "Surface plasmon resonance sensor based on planar light pipe: theoretical optimization analysis", Sensors and Actuators B, Chemical 37, 145-150 (1996).

[2] H. Raether, Surface Plasmons on Smooth and Rough Surfaces and on Gratings, chapter 2, Springer-Verlag, Berlin, 1988.

[3] S. A. Maier, Plasmonics: Fundamentals and Applications, chapter 2-3, Springer, Berlin, 2007.

[4] K. A. Peterlinz and R. Georgiadis, "Two-color approach for determination of thickness and dielectric constant of thin films using surface plasmon resonance spectroscopy", Optics Communications 130, 260-266 (1996).

[5] J. Homola, "Present and future of surface plasmon resonance biosensors", Analytical and Bioanalytical Chemistry 377, 528-539 (2003).

[6] J. Homola, S. S. Yee, and G. Gauglitz, "Surface plasmon resonance sensors: review", Sensors and Actuators B, Chemical 54, 3-15 (1999).

[7] M. Leitz, A. Tamachkiarow, H. Franke, and K. T. V. Grattan, "Monitoring of biofilm growth using ATR-leaky mode spectroscopy", Journal of Physics D: Applied Physics 35, 55-60 (2002).

[8] R. Ziblat, V. Lirtsman, D. Davidov, and B. Aroeti, "Infrared Surface Plasmon Resonance: A Novel Tool for Real Time Sensing of Variations in Living Cells", Biophysical Journal 90, 2592-2599 (2006).

[9] V. Lirtsman, M. Golosovsky, and D. Davidov, "Infrared surface plasmon resonance technique for biological studies", Journal of Applied Physics 103, 014702 (2008).

[10] R. L. Rich and D. G. Myszka, "Advances in surface plasmon resonance biosensor analysis", Current Opinion in Biotechnology 11, 54-61 (2000).

[11] B. K. Singh and A. C. Hillier, "Surface Plasmon Resonance Imaging of Biomolecular Interactions on a GratingBased Sensor Array", Analytical Chemistry 78, 2009-2018 (2006).

[12] M. Manuel, B. Vidal, R. López, S. Alegret, J. Alonso-Chamarro, I. Garces, and J. Mateo, "Determination of probable alcohol yield in musts by means of an SPR optical sensor", Sensors and Actuators B, Chemical 11, 455459 (1993).

[13] M. W. Foster, D. J. Ferrell, and R. A. Lieberman, "Surface plasmon resonance biosensor miniaturization", Proceedings of SPIE 2293, 122-131 (1994).

[14] J. Melendez, R. Carr, D. U. Bartholomew, K. Kukanskis, J. Elkind, S. Yee, C. Furlong, and R. Woodbury, "A commercial solution for surface plasmon sensing", Sensors and Actuators B, Chemical 35-36, 212-216 (1996).

${ }^{[15]}$ H. P. Ho, S. Y. Wu, M. Yang, and A. C. Cheung, "Application of white light-emitting diode to surface plasmon resonance sensors", Sensors and Actuators B, Chemical 80, 89-94 (2001).

[16] L. Bürgi, R. Pfeiffer, M. Mücklich, P. Metzler, M. Kiy, and C. Winnewisser, "Optical proximity and touch sensors based on monolithically integrated polymer photodiodes and polymer LEDs", Organic Electronics 7, 114-120 (2006).

[17] X. Wang, O. Hofmann, J. Huang, E. M. Barrett, R. Das, A. J. de Mello, J. C. de Mello, and D. D. C. Bradley, "Organic light emitting diodes and photodetectors: Toward applications in lab-on-a-chip portable devices", Proceedings of SPIE 6036, 603610 (2006).

${ }^{[18]}$ N. A. Reinke, J. Neumann, and W. Brütting, "Surface plasmon resonance spectroscopy device", patent pending, May 2007.

[19] P. Schiebener, J. Straub, J. M. H. Levelt Sengers, and J. S. Gallagher, "Refractive Index of Water and Steam as Function of Wavelength, Temperature and Density", Journal of Physical and Chemical Reference Data 19, 677-717 (1990).

[20] R. C. Weast (Ed.), CRC Handbook of Chemistry and Physics, 64th ed., pp. D-257-258, CRC Press, Inc., Boca Raton, 1983.

[21] I. M. White and X. Fan, "On the performance quantification of resonant refractive index sensors", Optics Express 16, 1020-1028 (2008). 
[22] R. Slavík and J. Homola, "Optical multilayers for LED-based surface plasmon resonance sensors", Applied Optics 45, 3752-3759 (2006).

[23] S. R. Karlsen, K. S. Johnston, S. S. Yee, and C. C. Jung, "First-order surface plasmon resonance sensor system based on a planar light pipe", Sensors and Actuators B, Chemical 32, 137-141 (1996). 